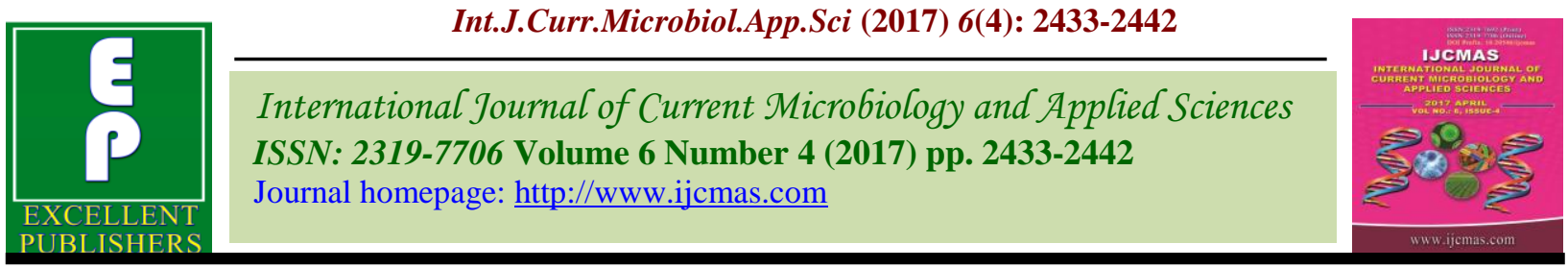

Original Research Article

https://doi.org/10.20546/ijcmas.2017.604.284

\title{
Discrimination of Calophyllum inophyllum L. Progenies Through ISSR Markers
}

\author{
B. Palanikumaran* and K.T. Parthiban \\ Department of Agroforestry, Forest College and Research Institute, Tamil Nadu Agricultural \\ University, Mettupalayam 641 301, India \\ *Corresponding author
}

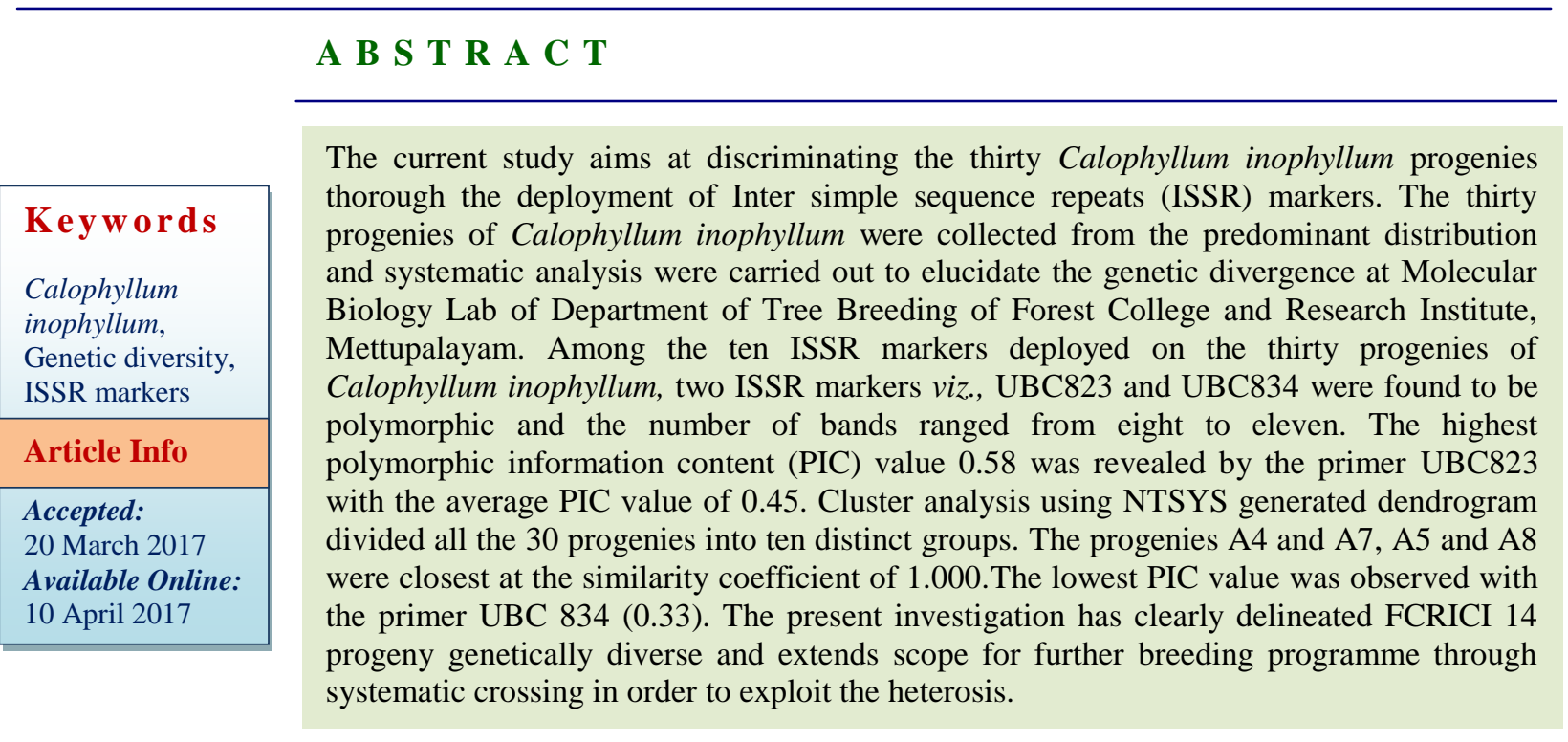

\section{Introduction}

Selection is the most important activity in all breeding programmes (Zobel and Talbert, 1984). Tree yield, i.e., tree volume is a complex and highly variable character which is influenced by many component characters (Deepak Pandey and Hooda, 1997). In the integrated structure of the plant, the overall correlation observed between two variables is a function of a series of direct and indirect relationships between different variables which could be affected through path coefficient studies (Wright, 1921). However, research pertaining to correlation and path analysis in Undi is very scant variable
(Divakara et al., 2010; Pavithra et al., 2013) and thus needs intensive research. The nature and degree of divergence in the base population is a pre-requisite for any improvement and conservation strategies (Gradual et al., 1999). The genetic diversity has been assessed traditionally either by provenance testing using Mahalanobis $\mathrm{D}^{2}$ statistics and Canonical vector analysis or electrophoretic analysis of enzymes. Study of metric characters in field trials was earlier the dominating technique and it is still today the most robust and valid way of assessing genetic variation. Markers also have 
important immediate application in supportive research for tropical hardwoods and nonindustrial species mainly for quantification of genetic diversity. There are several methods based on DNA markers viz., RFLP (Restriction Fragment Length Polymorphism), RAPD (Randomly Amplified Polymorphic DNA), SSR (Simple Sequence Repeats), ISSR (Inter Simple Sequence Repeats), AFLP (Amplified Fragment Length Polymorphism) etc. Among these techniques, ISSR marker is very significant because this marker in its simplest form, no prior genomic knowledge is required to design arbitrary sequence oligonucleotide primers (Gonzalez et al., 2005). But the use of molecular markers for genetic diversity studies in Undi dismally modest thus underscores the research needs.

\section{Materials and Methods}

The predominant Calophyllum inophyllum growing areas of Southern India viz., Tamil Nadu, Karnataka and Kerala were identified and Candidate Plus Trees were selected based on the following morphological features viz., Height, Diameter at Breast Height (DBH), and Canopy width by using the method described by Pitcher and Dorn (1966). From the selected 30 candidate plus trees seeds were collected from the crown and the morphometric attributes of selected candidate plus trees were presented in table 1 .

The ripened fruits of Calophyllum inophyllum were collected from the 30 selected candidate plus trees during the month of September and December. The 30 progenies were raised from the seeds collected from the selected candidate plus trees and One year old saplings were planted in the field by adopting Randomized Block Design.

Young leaf tissues were collected from 30 progenies of one year old saplings of Calophyllum inophyllum and the method followed to isolate genomic DNA was chloroform method as described by McCouch lab protocol (1998). The isolated genomic DNA was quantified using fluorometer (Dyna Quant, USA). The isolated DNA was verified for size, intactness, homogeneity and purity.

The isolated genomic DNA was subjected to polymerase chain reaction (PCR) using PERKIN ELMER Gene Amp PCR thermo cycler. The various 10 random ISSR primers viz., UBC808, UBC809, UBC810, UBC811, UBC823, UBC834, UBC835, UBC844, UBC848, UBC881 and UBC890 were used for DNA amplification are given in table 2 . Clear and unambiguous bands of ISSR markers were scored based on the presence or absence of the corresponding band among the genotypes. The scores ' 1 ' and ' 0 ' indicates the presence and absence of bands respectively. Statistical analysis was carried out by using the computer software NTSYSpc version 2.02 developed by Rohif (1998).

The confidence limits for the groupings by dendrograms were computed by using WINBOOT, a program for performing bootstrap analysis of binary data to determine the confidence limits of UPGMA based dendrograms (Yap and Nelson, 1996). Bootstrapping involves repeated sampling with replacement of the characters in matrix of operational taxonomic unit (OUT) $\mathrm{x}$ characters to create numerous bootstrap matrices of the same size as the original matrix.

From the binary code matrices obtained from the ISSR markers, the Jaccard's similarity indices Jaccard (1908) were computed for the 30 genotypes of Calophyllum inophyllum using the SIMQUAL programme of NTSYSpc version 2.02. Polymorphism information content (PIC) or expected heterozygosity scores for each ISSR marker was calculated as described by Anderson et al., (1993) 


\section{Results and Discussion}

Assessment of genetic diversity is a prerequisite for efficient conservation and utilization of genetic resources. During the past two decades, several high-throughput PCR-based technologies such as inter-simple sequence repeats (ISSR) and amplified fragment length polymorphisms (AFLP) have been developed to assay genetic polymorphism at the DNA level (Shyam Sundar et al.,2014).

An advantage of ISSRs is that in their simplest form, no prior genomic knowledge is required to design arbitrary sequence oligonucleotide primers (Gonzalez et al., 2005). With the large array of molecular analytical techniques available, it has become possible to provide an accurate and unambiguous tool for the evaluation of genetic diversity and identification of germplasm (Li et al., 2008).

Assessment of genetic variation within the species based on morphological and polypeptide descriptions, providing more speed, accurate and detailed information (Zabeau and Vos, 1993). ISSR has been used for diversity assessment by many researchers in tropical and temperate trees.

Extensive studies have already been reported in many tropical trees viz., Pongamia pinnata (Shyam Sundar et al., 2014); Casuarinas (Yasodha and Kathirvel, 2004) ISSR techniques was used to study the genetic structure and relationship between species and populations. Similarly, DNA based ISSR techniques have been successfully utilized in many genera and species of forest trees (Mohapatra and Singhal, 2000) for diversity study. In the present investigation, Out of 10
ISSR primers deployed, only 2primers viz., UBC 823 and UBC 834 exhibited high polymorphism (100 per cent) with all thirty genotypes of Calophyllum inophyllum. Nineteen reproducible bands were obtained from these two primers alone and the highest PIC value $(0.58)$ was recorded by the primer UBC823 and the lowest PIC value (0.33) was observed in the primer UBC834 (Table 3).

The present investigation revealed that Undiaccessions used for diversity assessment through ISSR, showed moderate level of genetic variations. Polymorphism in several tree species was documented by several authors viz., Jatropha spp. (Sunil kumar et al., 2013); and Tamarindus indica (Nandini et al., 2011). PCR-based DNA markers were employed to assess genetic diversity in Jatropha curcas genotypes (Jubera et al., 2009) and Casuarina equisetifolia (Elavazhagan et al., 2009).

The study with DNA markers across species of Jatropha has revealed a high level of genetic diversity (Basha et al., 2009). In Undi, the average linkage between the genotypes was used for constructing a phylogenetic tree. The association amongst different genotypes was presented in the form of dendrogram (Fig. 1). The progenies A4 and A7, A5 and A8, were closest at the similarity coefficient 1.000 . The progenies A1 and A2, A2 and A19, A2 and A20, $\mathrm{A} 2$ and $\mathrm{A} 25, \mathrm{~A} 5$ and $\mathrm{A} 15, \mathrm{~A} 8$ and $\mathrm{A} 15, \mathrm{~A} 12$ and A28 were the farthest or most diverse and dissimilar to all others (Table 4).

Similar grouping pattern within species and related genotypes are evidenced from the earlier reports in Eucalyptus tereticornis (Suman Tewari et al., 2013). 
Table.1 Morphological characters of selected superior progenies of Calophyllum inophyllum

\begin{tabular}{|c|c|c|c|c|}
\hline SI. No. & $\begin{array}{c}\text { Name of the } \\
\text { CPTs }\end{array}$ & $\begin{array}{c}\text { Height } \\
(\mathbf{m})\end{array}$ & $\begin{array}{c}\text { GBH } \\
(\mathbf{m})\end{array}$ & $\begin{array}{c}\text { Crown } \\
\text { diameter } \\
(\mathbf{m})\end{array}$ \\
\hline 1 & FCRICI 1 & 4.31 & 0.71 & 3.07 \\
2 & FCRICI 2 & 5.50 & 0.83 & 2.95 \\
3 & FCRICI 3 & 4.16 & 0.76 & 2.67 \\
4 & FCRICI 4 & 5.34 & 0.97 & 3.21 \\
5 & FCRICI 5 & 5.02 & 0.75 & 4.02 \\
6 & FCRICI 6 & 5.96 & 0.59 & 3.26 \\
7 & FCRICI 7 & 4.25 & 0.65 & 4.50 \\
8 & FCRICI 8 & 5.93 & 0.73 & 2.79 \\
9 & FCRICI 9 & 5.14 & 0.82 & 3.08 \\
10 & FCRICI 10 & 5.96 & 0.70 & 2.99 \\
11 & FCRICI 11 & 5.08 & 0.67 & 3.45 \\
12 & FCRICI 12 & 4.13 & 0.72 & 2.13 \\
13 & FCRICI 13 & 4.66 & 0.69 & 3.05 \\
14 & FCRICI 14 & 7.98 & 1.02 & 5.67 \\
15 & FCRICI 15 & 6.97 & 0.83 & 4.97 \\
16 & FCRICI 16 & 7.02 & 0.90 & 4.06 \\
17 & FCRICI 17 & 7.89 & 0.79 & 3.96 \\
18 & FCRICI 18 & 6.48 & 0.84 & 4.56 \\
19 & FCRICI 19 & 7.36 & 0.92 & 2.13 \\
20 & FCRICI 20 & 8.01 & 0.86 & 3.20 \\
21 & FCRICI 21 & 7.00 & 0.64 & 4.16 \\
22 & FCRICI 22 & 6.71 & 0.59 & 3.99 \\
23 & FCRICI 23 & 6.13 & 0.96 & 3.54 \\
24 & FCRICI 24 & 5.67 & 0.95 & 2.87 \\
25 & FCRICI 25 & 5.95 & 0.66 & 2.40 \\
26 & FCRICI 26 & 6.03 & 0.76 & 4.88 \\
27 & FCRICI 27 & 4.05 & 0.80 & 2.97 \\
28 & FCRICI 28 & 3.21 & 0.50 & 2.18 \\
29 & FCRICI 29 & 5.69 & 0.72 & 3.22 \\
30 & FCRICI 30 & 5.01 & 0.65 & 2.43 \\
\hline & Mean & $\mathbf{5 . 7 5}$ & $\mathbf{0 . 7 6}$ & $\mathbf{3 . 4 1}$ \\
\hline
\end{tabular}


Int.J.Curr.Microbiol.App.Sci (2017) 6(4): 2433-2442

Table.2 List of primers used for ISSR amplification

\begin{tabular}{|c|c|c|}
\hline S.No & Primers & Primer sequence 5'-3', \\
\hline 1. & UBC808 & AGAGAGAGAGAGAGAGC \\
\hline 2. & UBC809 & AGAGAGAGAGAGAGAGG \\
\hline 3. & UBC810 & GAGAGAGAGAGAGAGAT \\
\hline 4. & UBC811 & GAGAGAGAGAGAGAGAC \\
\hline 5. & UBC823 & ТСТСТСТСТСТСТСТСС \\
\hline 6. & UBC834 & AGAGAGAGAGAGAGAGYT \\
\hline 7. & UBC835 & AGAGAGAGAGAGAGAGYC \\
\hline 8. & UBC844 & СТСТСТСТСТСТСТСТRС \\
\hline 8. & UBC848 & CACACACACACACACARG \\
\hline 9. & UBC881 & GGGTGGGGTGGGGTG \\
\hline 10. & UBC890 & VHVGTGTGTGTGTGTGT \\
\hline
\end{tabular}

Table.3 Random ISSR primers used for DNA amplification in Calophyllum inophyllum

\begin{tabular}{|c|c|c|c|c|}
\hline $\begin{array}{c}\text { Sl. } \\
\text { No }\end{array}$ & \multirow{2}{*}{ Primers } & Sequence 5'-3, & Total number of bands & PIC values \\
\hline 1 & UBC823 & TCTCTCTCTCTCTCTCC & 11 & 0.58 \\
2 & UBC834 & AGAGAGAGAGAGAGAGYT & 08 & 0.33 \\
\hline \multicolumn{2}{|c|}{ Mean } & $\mathbf{0 . 4 5}$ \\
\hline
\end{tabular}


Table.4 ISSR Similarity matrix of Calophyllum inophyllum progenies

\begin{tabular}{|c|c|c|c|c|c|c|c|c|c|c|c|c|c|c|c|c|c|c|c|c|c|c|c|c|c|c|c|c|c|c|}
\hline Seed & 1 & 2 & 3 & 4 & 5 & 6 & 7 & 8 & 9 & 10 & 11 & 12 & 13 & 14 & 15 & 16 & 17 & 18 & 19 & 20 & 21 & 22 & 23 & 24 & 25 & 26 & 27 & 28 & 29 & 30 \\
\hline 1 & 1.0000 & & & & & & & & & & & & & & & & & & & & & & & & & & & & & \\
\hline 2 & 0.3157 & \begin{tabular}{|l|l|}
1.0000 \\
\end{tabular} & & & & & & & & & & & & & & & & & & & & & & & & & & & & \\
\hline 3 & 0.5789 & 0.5263 & 1.0000 & & & & & & & & & & & & & & & & & & & & & & & & & & & \\
\hline 4 & 0.4210 & \begin{tabular}{|l|l|}
0.8947 \\
\end{tabular} & 0.6315 & .0000 & & & & & & & & & & & & & & & & & & & & & & & & & & \\
\hline 5 & 0.3684 & 0.8421 & 0.5789 & .9473 & 31.0000 & & & & & & & & & & & & & & & & & & & & & & & & & \\
\hline 6 & 0.4736 & 0.8421 & 0.4736 & .8421 & \begin{tabular}{l|l|}
0.7894 \\
\end{tabular} & 1.0000 & & & & & & & & & & & & & & & & & & & & & & & & \\
\hline 7 & 0.4210 & 0.8947 & 0.6315 & .0000 & 0.9473 & 0.8421 & 1.0000 & & & & & & & & & & & & & & & & & & & & & & & \\
\hline 8 & $\mid 0.3684$ & 0.8421 & 0.5789 & .9473 & $\begin{array}{ll}31.0000 \\
\end{array}$ & $\mid 0.7894$ & 0.9473 & \begin{tabular}{l|l}
3 & 1.0000
\end{tabular} & & & & & & & & & & & & & & & & & & & & & & \\
\hline 9 & 0.4736 & \begin{tabular}{|l|l|} 
\\
\end{tabular} & 0.5789 & .5263 & 0.4736 & \begin{tabular}{|l|}
0.5789 \\
\end{tabular} & 0.5263 & 30.4736 & 1.0000 & & & & & & & & & & & & & & & & & & & & & \\
\hline 10 & 0.5789 & 0.6315 & 0.7894 & .7368 & 0.6842 & 0.5789 & 0.7368 & \begin{tabular}{|l|l|}
8.6842 \\
\end{tabular} & 0.7894 & 1.0000 & & & & & & & & & & & & & & & & & & & & \\
\hline 11 & 0.4736 & 0.6315 & 0.6842 & .7368 & 0.7894 & \begin{tabular}{|l|l|} 
\\
\end{tabular} & 0.7368 & 80.7894 & 0.68420 & 0.8947 & 1.0000 & & & & & & & & & & & & & & & & & & & \\
\hline 12 & 0.4210 & 0.6842 & 0.4210 & .7894 & 0.8421 & 0.6315 & 0.7894 & 40.8421 & 0.42100 & 0.6315 & 0.7368 & 1.0000 & & & & & & & & & & & & & & & & & & \\
\hline 13 & 0.6315 & 0.4736 & 0.6315 & .4736 & 0.4210 & 0.5263 & 0.4736 & 6.4210 & 0.73680 & 0.7368 & 0.6315 & 0.5789 & 1.0000 & & & & & & & & & & & & & & & & & \\
\hline 14 & 0.6315 & 0.5789 & 0.7368 & .6842 & 0.6315 & 0.6315 & 0.6842 & $\mid 0.6315$ & 0.63150 & 0.7368 & 0.6315 & 0.5789 & 0.7894 & 1.0000 & & & & & & & & & & & & & & & & \\
\hline 15 & $\mid 0.5263$ & \begin{tabular}{|l|l|}
0.3684 \\
\end{tabular} & 0.7368 & .3684 & $\begin{array}{l}40.3157 \\
\end{array}$ & 0.4210 & 0.3684 & $4 \mid 0.3157$ & 0.73680 & 0.6315 & 0.5263 & 0.3684 & 0.7894 & 0.6842 & 1.0000 & & & & & & & & & & & & & & & \\
\hline 16 & 0.6842 & 0.5263 & 0.7894 & .6315 & 0.5789 & 0.5789 & 0.6315 & $5 \mid 0.5789$ & 0.68420 & 0.7894 & 0.6842 & 0.52630 & 0.8421 & 0.9473 & 0.7368 & 1.0000 & & & & & & & & & & & & & & \\
\hline 17 & $\mid 0.5263$ & 0.6842 & 0.6315 & .7894 & 0.7368 & 0.7368 & 0.7894 & $4 \mid 0.7368$ & 0.52630 & 0.6315 & 0.6315 & 0.68420 & 0.6842 & 0.8947 & 0.5789 & 0.8421 & \begin{tabular}{|l|l|}
1.0000 \\
\end{tabular} & & & & & & & & & & & & & \\
\hline 18 & 0.6315 & 0.5789 & 0.6315 & .6842 & 0.6315 & 0.6315 & 0.6842 & 0.6315 & 0.52630 & 0.6315 & 0.6315 & 0.5789 & 0.6842 & 0.8947 & 0.5789 & 0.8421 & 0.8947 & 1.0000 & & & & & & & & & & & & \\
\hline 19 & $|0.6842|$ & 0.3157 & 0.7894 & .4210 & 0.4736 & 0.3684 & 0.4210 & $\mid 0.4736$ & 0.4736 & 0.5789 & 0.5789 & 0.4210 & 0.6315 & 0.7368 & 0.7368 & 0.7894 & 0.6315 & 0.7368 & 1.0000 & & & & & & & & & & & \\
\hline 20 & $\mid 0.5789$ & 0.3157 & 0.6842 & .4210 & 0.4736 & 0.3684 & 0.4210 & 0.4736 & 0.36840 & 0.5789 & 0.5789 & 0.5263 & 0.5263 & 0.6315 & 0.6315 & 0.5789 & 0.5263 & 30.6315 & 0.7894 & 1.0000 & & & & & & & & & & \\
\hline 21 & 0.6315 & 0.4736 & 0.7368 & .5789 & 0.5263 & 0.5263 & 0.5789 & 90.5263 & 0.31570 & 0.5263 & 0.5263 & 0.4736 & 0.4736 & 0.6842 & 0.5789 & 0.6315 & 0.6842 & 0.7894 & 0.7368 & 0.8421 & 1.0000 & & & & & & & & & \\
\hline 22 & $\mid 0.6842$ & 0.5263 & 0.6842 & .6315 & 50.5789 & \begin{tabular}{|l|}
0.5789 \\
\end{tabular} & 0.6315 & $5 \mid 0.5789$ & 0.47360 & 0.5789 & 0.5789 & 0.5263 & 0.5263 & 0.7368 & 0.6315 & 0.6842 & 0.7368 & $3 \mid 0.8421$ & 0.7894 & 0.6842 & 0.8421 & 1.0000 & & & & & & & & \\
\hline 23 & $\mid 0.4736$ & 0.5263 & 0.6842 & .6315 & 0.6842 & $\mid 0.5789$ & 0.6315 & $5|0.6842|$ & 0.36840 & 0.4736 & 0.4736 & 0.6315 & 0.5263 & 0.7368 & 0.6315 & 0.6842 & 0.7368 & $\mid 0.6315$ & 0.7894 & 0.6842 & 0.6315 & 0.6842 & 1.0000 & & & & & & & \\
\hline 24 & $0.6315 \mid$ & 0.4736 & 0.8421 & .5789 & 0.5263 & 0.5263 & 0.5789 & \begin{tabular}{|l|l|}
9 & 0.5263 \\
\end{tabular} & 0.52630 & 0.6315 & 0.6315 & 0.4736 & 0.6842 & 0.7894 & 0.7894 & 0.8421 & \begin{tabular}{|l|l|}
0.7894 \\
\end{tabular} & 0.7894 & 0.8421 & 0.6315 & 0.7894 & 0.8421 & \begin{tabular}{|l|l|}
0.73681 \\
\end{tabular} & 1.0000 & & & & & & \\
\hline 25 & $\mid 0.7894$ & 0.3157 & 0.5789 & .4210 & 0.3684 & 0.3684 & 0.4210 & $0.3684 \mid$ & 0.36840 & 0.4736 & 0.3684 & 0.4210 & 0.6315 & 0.6315 & 0.5263 & 0.6842 & 0.6315 & 0.6315 & 0.6842 & 0.4736 & 0.5263 & 30.5789 & 0.57890 & 0.6315 & 1.0000 & & & & & \\
\hline 26 & $\mid 0.5263$ & 0.5789 & 0.6315 & 1.6842 & 0.6315 & 0.6315 & 0.6842 & 0.6315 & 0.63150 & 0.7368 & 0.7368 & 0.68420 & 0.7894 & 0.8947 & 0.68420 & 0.8421 & \begin{tabular}{|l|l|}
0.8947 \\
\end{tabular} & 0.8947 & 0.6315 & 50.6315 & 0.6842 & 0.7368 & 0.63150 & 0.7894 & 0.5263 & \begin{tabular}{|l|l|}
1.0000 \\
\end{tabular} & & & & \\
\hline 27 & $\mid 0.4736$ & 0.5263 & 0.6842 & .6315 & 0.5789 & \begin{tabular}{|l|}
0.5789 \\
\end{tabular} & 0.6315 & $5|0.5789|$ & 0.57890 & 0.6842 & 0.6842 & 0.63150 & 0.7368 & 0.8421 & 0.7368 & 0.7894 & \begin{tabular}{|l|l|}
0.8421 \\
\end{tabular} & $\mid 0.8421$ & 0.6842 & 0.6842 & 0.7368 & 0.7894 & 0.68420 & 0.8421 & $\mid 0.4736$ & \begin{tabular}{|l|}
0.9473 \\
\end{tabular} & \begin{tabular}{|l|l|l}
3.0000 \\
\end{tabular} & & & \\
\hline 28 & 0.4736 & 0.5263 & 0.6842 & .4210 & 0.3684 & 0.4736 & 0.4210 & $\mid 0.3684$ & 0.68420 & 0.4736 & 0.3684 & 0.3157 & 0.6315 & 0.7368 & 0.84210 & 0.6842 & 0.6315 & 0.6315 & 0.6842 & 0.5789 & 0.6315 & 0.6842 & 0.68420 & 0.7368 & 0.4736 & 0.6315 & 0.68421 & 1.0000 & & \\
\hline 29 & 0.5789 & 0.4210 & 0.7894 & .5263 & \begin{tabular}{l|l}
3 & 0.4736
\end{tabular} & 0.4736 & 0.5263 & 30.4736 & 0.57890 & 0.6842 & 0.5789 & 0.5263 & 0.7368 & 0.8421 & $0.8421 \mathrm{C}$ & 0.7894 & 0.7368 & 0.7368 & 0.7894 & 0.7894 & 0.7368 & 0.7894 & 0.7894 & 0.8421 & 0.5789 & 0.8421 & 0.89470 & 0.7894 & 1.0000 & \\
\hline 30 & 0.5263 & 0.5789 & 0.6315 & .5789 & 0.5263 & 0.6315 & 0.5789 & 90.5263 & 0.7368 & .7368 & 0.7368 & 0.5789 & 0.8947 & 0.7894 & 0.7894 & 0.8421 & 0.7894 & 0.7894 & 0.6315 & 0.5263 & 0.5789 & 0.6315 & 0.52630 & 0.7894 & 0.5263 & 0.8947 & 0.84210 & 0.63150 & 0.7368 & 1.0000 \\
\hline & & 1 & & & CRICI 1 & & & & & & 11 & & FCRII & CI 11 & & & & & & 21 & & FCRICI & & & & & & & & \\
\hline & & 2 & & & CRICI 2 & & & & & & 12 & & FCRIC & CI 12 & & & & & & 22 & & FCRICI & & & & & & & & \\
\hline & & 3 & & & CRICI 3 & & & & & & 13 & & FCRIC & CI 13 & & & & & & 23 & & FCRICI & & & & & & & & \\
\hline & & 4 & & & CRICI 4 & & & & & & 14 & & FCRIC & CI 14 & & & & & & 24 & & FCRICI & & & & & & & & \\
\hline & & 5 & & & CRICI 5 & & & & & & 15 & & FCRIC & CI 15 & & & & & & 25 & & FCRICI & & & & & & & & \\
\hline & & 6 & & & CRICI 6 & & & & & & 16 & & FCRIC & CI 16 & & & & & & 26 & & FCRICI & & & & & & & & \\
\hline & & 7 & & & CRICI 7 & & & & & & 17 & & FCRIC & CI 17 & & & & & & 27 & & FCRICI & & & & & & & & \\
\hline & & 8 & & & CRICI 8 & & & & & & 18 & & FCRIC & CI 18 & & & & & & 28 & & FCRICI & & & & & & & & \\
\hline & & 9 & & & CRICI 9 & & & & & & 19 & & FCRIC & CI 19 & & & & & & 29 & & FCRICI & & & & & & & & \\
\hline & & 10 & & & CRICI 10 & & & & & & 20 & & FCRIC & CI 20 & & & & & & 30 & & FCRICI & & & & & & & & \\
\hline
\end{tabular}




\section{UBC823 (TCTCTCTCTCTCTCTCC)}

$\begin{array}{lllllllllllllllllllllllllllllll}M & 1 & 2 & 3 & 4 & 5 & 6 & 7 & 8 & 9 & 10 & 11 & 12 & 13 & 14 & 15 & 16 & 17 & 18 & 19 & 20 & 21 & 22 & 23 & 24 & 25 & 26 & 27 & 28 & 29 & 30\end{array}$

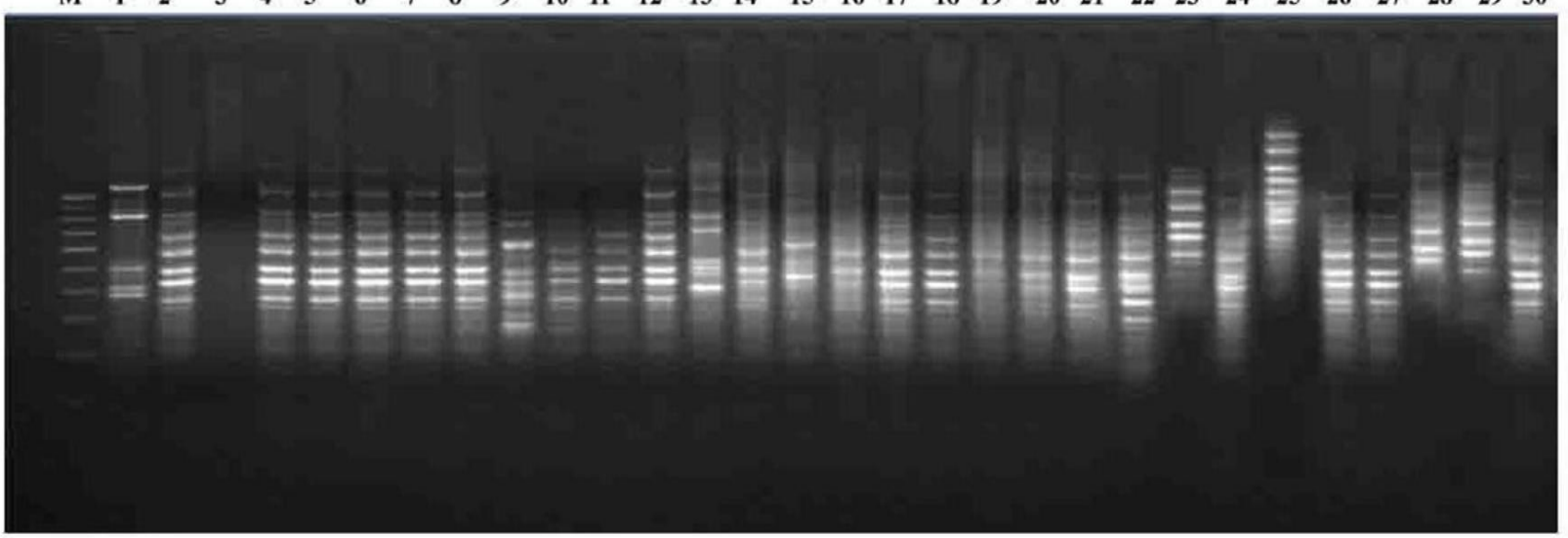

UBC834 (AGAGAGAGAGAGAGAGYT)

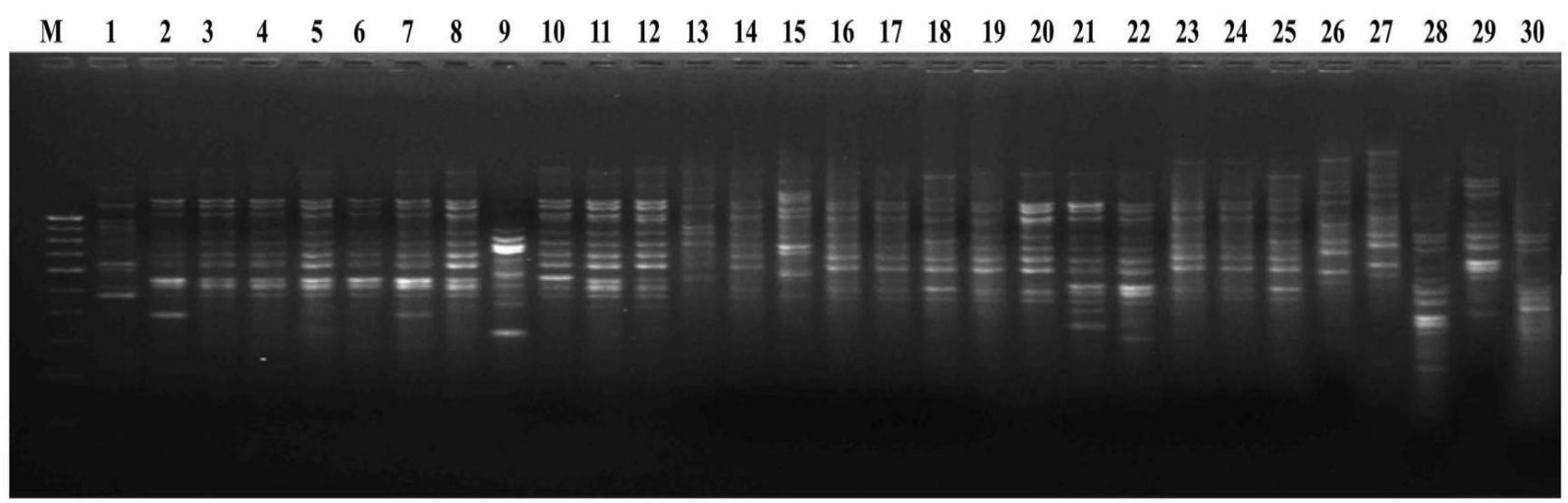


Fig.2 Dendrogram of Calophyllum inophyllum progenies based on Jaccard's similarity coefficient

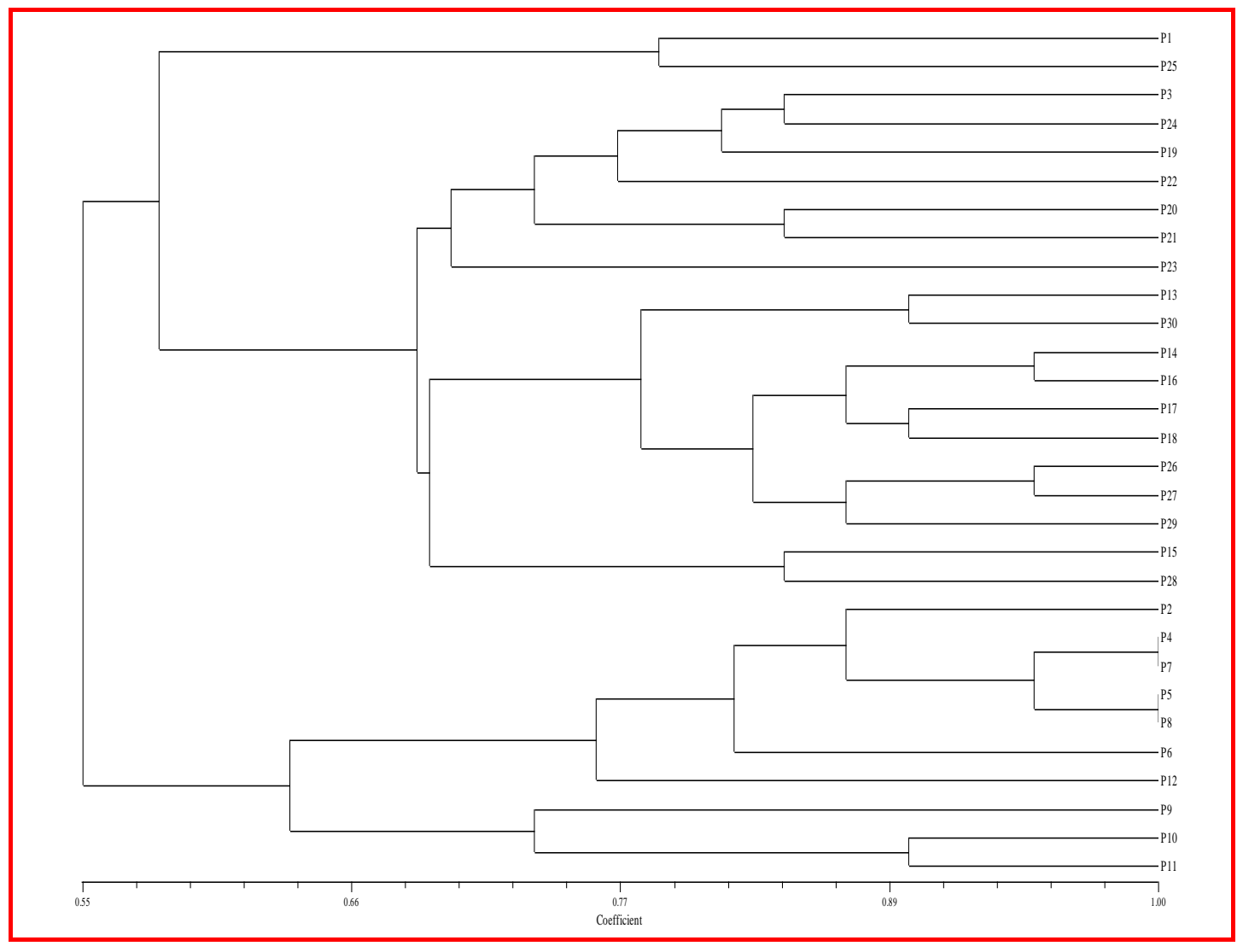

P1- FCRICI 1 P4- FCRICI 4 P7- FCRICI 7 P10- FCRICI 10 P13- FCRICI 13 P16- FCRICI 16 P19- FCRICI 19 P22- FCRICI 22 P25- FCRICI 25 P28- FCRICI 28 P2-FCRICI 2 P5- FCRICI 5 P8- FCRICI 8 P11- FCRICI 11 P14- FCRICI 14 P17- FCRICI 17 P20- FCRICI 20 P23- FCRICI 23 P26- FCRICI 26 P29- FCRICI 29 P3- FCRICI 3 P6- FCRICI 6 P9- FCRICI 9 P12- FCRICI 12 P15- FCRICI 15 P18- FCRICI 18 P21- FCRICI 21 P24- FCRICI 24 P27- FCRICI 27 P30- FCRICI 30 
The results revealed that ISSR is an efficient technique to characterize the Undigenotypes and classify different genotypes based on the ISSR markers generated (Fig. 2). It was also indicated that ISSR analysis has determined the genetic relationships and estimated the genetic diversity among the genotypes of Undi. The results of the present study can be used as a stepping stone for evolving well defined approach based on evaluation and characterization of genetic variation in Undi genotypes which can be further used for the improvement of these two species for various traits through different breeding methods.

In conclusion the molecular analysis of Undi progenies through ISSR markers identified the progenies A4 and A7 and A5 and A8 exhibited closest similarity coefficient. In a holistic prospection, the current investigation identified one superior progeny each in Undi (FCRICI 14)for further breeding programme through systematic crossing in order to exploit the heterosis.

\section{References}

Anderson, J.A., G.A. Churchill, J.E. Autrique, S.D. Tanksley and M.E. Sorrells. 1993. Optimizing parental selection for genetic linkage maps. Genome, 36: 181-186.

Basha, S.D., H.P.S. George Francis, K. Makkar, M. Becker and M. Sujatha. 2009. A comparative study of biochemical traits and molecular markers for assessment of genetic relationships between Jatropha curcas L. germplasm from different countries. Plant Science, 176: 812823.

Deepak Pandy and M.S. Hooda. 1997. Genetic variability and correlation studies in seed traits of mesquite (Prosopis juliflora (SW) DC.). Indian J. For.,24(2): 162-165.
Divakara, B.N., A.S. Alur and S. Tripathi. 2010. Genetic variability and relationship of pod and

seed traits in Pongamia pinnata (L.) Pierre., a potential agroforestry tree. International Journal of Plant Production, 4(2): 129-14.

Elavazhagan, T., M. Ramakrishnan, S. Jayakumar, C. Chitravadivu and V. Balakrishnan. 2009. DNA finger printing analysis in Casuarina equisetifolia by using RAPD markers. Botany Research International, 2(4): 244-247.

Gonzalez, A., A. Wong, A. Delgado-Salinas, R. Papa and P. Grepts. 2005. Assessment of inter simple sequence repeat markers to differentiate sympatric wild and domesticated populations of common bean. Crop Science,45(2): 606-615.

Gradual, L., E.D. Kjaer, P. Suangtho and A. Kaosaard. 1999. Conservation of genetic resources of teak (Tectona grandis) in Thailand. Technical Note No. 52. Danida Forest Seed Centre, Denmark.

Haines, R.J. and B.E. Martin. 1995. Biotechnology and the sustainable production of tropical timber. ITTO Pre-Project Report PPR 42 /97(F): 168.

Jaccard, P. 1908.Nouvellesrecherchessur distribution florale. Bull. Soc. Vaud. Nat., 44: 223-270.

Jubera, B.S., D.P. Janagoudar, R.L. Biradar, R. Ravikumar, V. Koti and S.J. Patil. 2009. Genetic diversity analysis of elite Jatropha curcas(L.) genotypes using Randomly Amplified Polymorphic DNA markers. Karnataka J. Agric. Sci., 22 (2): $293-$ 295.

Li, F., S. Gan, Q. Weng, X. Zhao, S. Huang, M. Li, S. Chen, Q. Wang and F. Shi. 2008. RAPD and morphological 
diversity among four populations of the tropical tree species Paramichelia baillonii (Pierre) $\mathrm{Hu}$ in China. Forest Ecology and Management,255: 17931801.

Mohapatra and Singhal. 2000. Role of molecular markers in biodiversity conservations. Ann. For., 8(1): 1-7.

Nandini, R., T.R. Singh and G.N. Dhanapal. 2011. Morphometric and molecular diversity studies in Tamarindus indica population. Plant Molecular Biology and Biotechnology, 2(2): 26-33.

Pavithra, H.M., B. Gowda, K.T. Prasanna and M.B. Shivanna. 2013. Pod and seed traits in candidate plus trees of Pongamia pinnata from southern peninsular India in relation to provenance variation and genetic variability. Korean Society of Crop Science: 012-0052-8.

Rohlf, F.J. 2002. NTSYS-pc: Numerical taxonomy system ver. 2.1. Exeter Publishing Ltd., Setauket, New York.

Sharp, P.A., B. Sugden and J. Sambrook. 1973. Detection of two restriction endonuclease activities Shyamsundar, S., K. Aadil, M.S. Negi and Tripathi. 2014. Efficiency of two dominant marker system, ISSR and TE-AFLP for assessment of genetic diversity in biodiesel species Pongamia pinnata. Current Science, 106:1576-1580.

SumanTiwari, RakhiTiwari, RichaSrivastava, PratibhaKumari and Sujeet Kr. Singh. 2013.

Molecular genetic analysis of Eucalyptus tereticornis by using RAPD markers. European Journal of Experimental Biology, 3(6): 103-110.

Sunil Kumar, Virendra Kumar, Manoj Kumar Sharma, Narendra Kumar, Amit Kumar, R.S. Sengar and NidhiJaiswal. 2013. Assessment of genetic diversity in Jatropha (Jatropha curcas L.) by using RAPD markers. International Journal of Biotechnology and Bioengineering Research, 4(4): 383394.

Williams, J.G.K., A.R. Kubelik, K.J. Livak, J.A. Rafalski and S.V. Tingey. 1990. DNApolymorphisms amplified by arbitrary primers are useful as genetic markers. Nucleic Acids Res., 18:65316535.

Wright, S. 1921. Correlation and causation. J. Agric. Res., 20: 557-585.

Yap, I.V. and R.J. Nelson. 1996. WINBOOT: a program for performing bootstrap analysis for

binary data to determine the confidence limits of UPGMA-based dendrograms. IRRI Disc. Ser. No. 14. Int. Rice Res. Inst., Manila, Philippines.

Yasodha, R. and M. Kathirvel. 2004. Genetic analyses of Casuarinas using ISSR and FISSR markers. Genetica, 122(2): 161-172.

Zabeau, M. and P. Vos. 1993. Selective restriction fragment amplification: a general method for DNA fingerprinting. European Patent.

Zobel, B.J. and J. Talbert. 1984. Applied tree improvement. John Wiley \& Co., New York. 503 p.

\section{How to cite this article:}

Palanikumaran, B. and Parthiban, K.T. 2017. Discrimination of Calophyllum inophyllum L. Progenies Through ISSR Markers. Int.J.Curr.Microbiol.App.Sci. 6(4): 2433-2442. doi: https://doi.org/10.20546/ijcmas.2017.604.284 